\title{
Tn917 Transposase. Sequence Correction Reveals a Single Open Reading Frame Corresponding to the tnpA Determinant of Tn3-Family Elements
}

\author{
Florence Y. AN* AND DON B. Clewell*,† \\ *Department of Biologic and Materials Sciences, School of Dentistry, and $\dagger$ Department of Microbiology \\ and Immunology, School of Medicine, The University of Michigan, Ann Arbor, Michigan 48109
}

Received October 9, 1990; revised January 22, 1991

\begin{abstract}
A nucleotide sequence correction on the Enterococcus faecalis transposon $\operatorname{Tn} 917$ indicates that what was formerly thought to be two open reading frames (ORF5 and ORF6) is actually one reading frame (ORF5). The latter exhibits homology with the Tn3-family transposase determinants known as $t n p A$. $(0) 1991$ Academic Press, Inc.
\end{abstract}

$\operatorname{Tn} 917$ was originally identified on the plasmid pAD2 in the multiply antibiotic resistant Enterococcus faecalis strain DS16 (Tomich et al., 1979, 1980). It encodes an inducible MLS (macrolide, lincosamide, and streptogramin B) resistance and is a member of the Tn3-family of transposons (Heffron, 1983; Sherratt, 1989). There is a close general resemblance to the staphylococcal transposon Tn551 (Perkins and Youngman, 1984; Khan and Novick, 1980), except that the latter expresses MLS resistance constitutively.

The complete nucleotide sequence of Tn917 has been previously reported (Shaw and Clewell, 1985). Three open reading frames were found to be associated with transposition functions. One of these, referred to as ORF4, was found to exhibit homology with Tn3-family resolvase determinant (tnpR). The other two open reading frames, ORF5 and ORF6, appeared to be associated with transposase activity (Pcrkins and Youngman, 1984; Shaw and Clewell, 1985). Although homology of the latter two reading frames with $\operatorname{Tn} 3$-family transposase $(\operatorname{tnp} A)$ was not noted, subsequent computer comparisons indicated significant homology indeed existed (Murphy, 1989). This prompted a re-

The sequence data from this article has been deposited with the EMBL/GenBank Data Libraries under Accession No. M36722. examination of this region of the transposon, the results of which are reported here. Correction of sequencing errors (mainly frameshiftrelated) has revealed that ORF5 and ORF6 are actually combined and represent a single ORF (now ORF5 or $t n p A$ ).

Figure 1 shows the corrected sequence of tnpA. The protein product is inferred to consist of 973 amino acid residues and to have a molecular weight of 112,205 . This compares favorably with the tnpA products of Tn 3 family elements (Sherratt, 1989). The $3^{\prime}$ end of the reading frame is $17 \mathrm{bp}$ from an inverted repeat segment (positions 3098 through 3140) which would form a stem consisting of $16 \mathrm{bp}$ with an 11-bp intervening loop ( $\Delta G$ is $-25.4 \mathrm{kcal}$ [Tinoco et al., 1973]). The latter might correspond to a transcription terminator; although subsequent multiple T's, generally associated with $\rho$-independent transcription terminators, are not present.

The corrections and their positions werc as follows: $\mathrm{G}$ converted to $\mathrm{C}$ at position 687 ; insert $A$ at 1803; insert $G$ at 1804; delete $G$ between 1807 and 1808; delete $T$ between 1905 and 1906; insert $G$ at 2186; insert $G$ at 2809 ; insert $T$ at 3079 ; insert $G$ at 3082 .

Figure 2 presents a homology matrix relating to the tnpA products of Tn501 (Brown et al., 1985), Tn21 (Ward and Grinsted, 1987), and $\operatorname{Tn} 3$ (Heffron et al., 1979); significant homology is clearly evident. The degrees of homology (identical residues) are 32, 32, and 


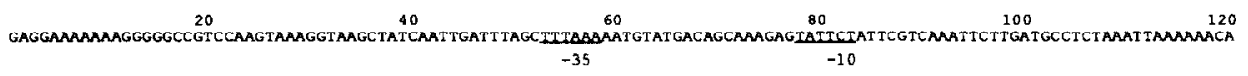

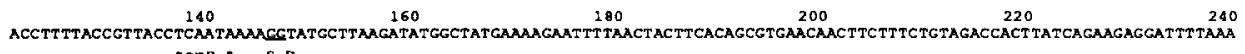
tIPR " S.D. $\quad$ M A GCGTATTTTAGTTTTTCTGATTATGATCTGGAGGTTTTTAATCAACACCGTGGAAAGGTCAATAAACTAGGATTTGCGATACAACTTTGTTTGGCCCGGTATCCTGGGTGTTCTTTAAGT

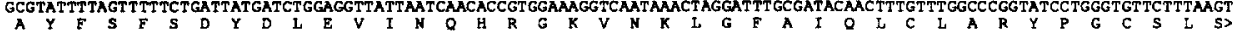

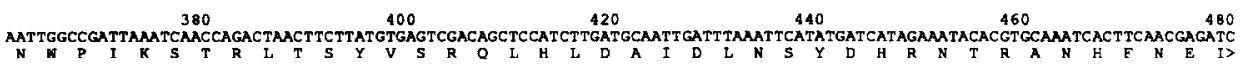

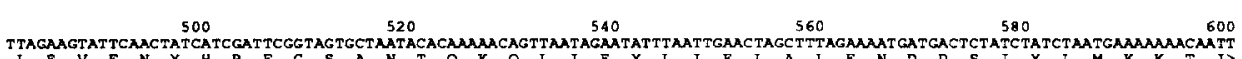
$\begin{array}{rllll}620 & 640 & 660 & 680 & 700\end{array}$

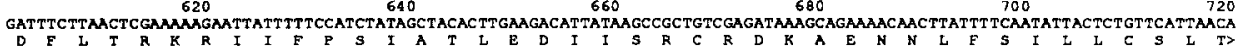

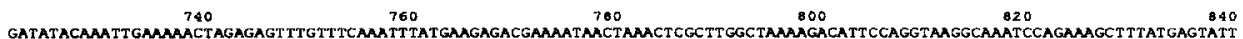

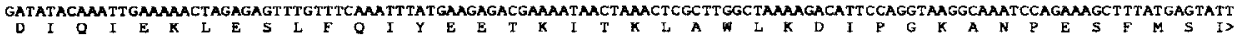
TGTAAMMAGTGGAAGTGATTGCTTCCATGGGACTCGGGACAATTAATGTCTCCCATATTAATCGGAACAGGTTTCTTCAGCTAGCTAGACTAGGGGAAAATTATGATGCATATGACTTC $\underset{C}{\text { TGTAAMMAGTGGAAGTGATTGCTTCCATGGGACTCGGGACAATTAATGTCTCCCATATTAATCGGAACAGGTTTCTTCAGCTAGCTAGACTAGGGGAAAATTATGATGCATATGACTTC }}$

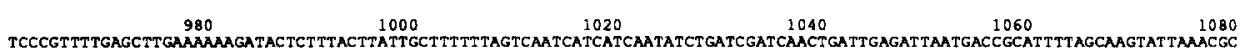

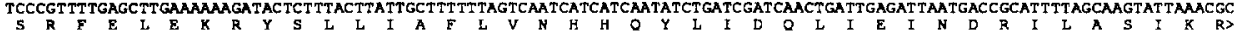

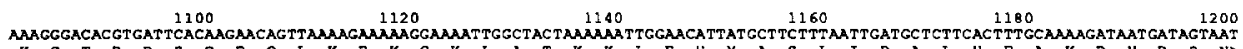

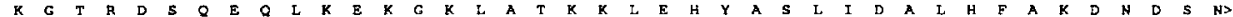
CCTTTTGACGAATtGaA $1220 \quad 1240 \quad 1260 \quad 1280 \quad 1300$

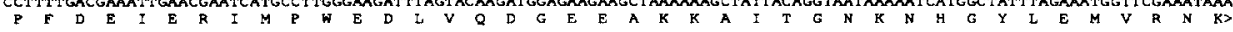

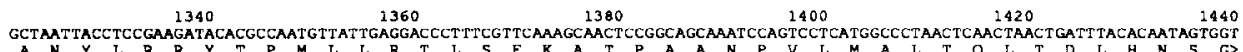
$1460 \quad 15800001520 \quad 1540 \quad 1560$

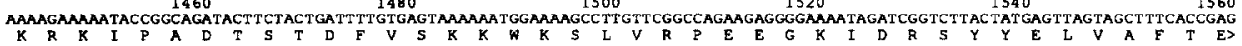
$\begin{array}{llllll}1580 & 1600 & 1620 & 1640 & 1660 & 1680\end{array}$ CTAAAGACAATATT CGATCAGGAGATATTTCAGTTGAAGGAGTATGATCCATCGAATATTGATGATTACTTAGTTGATTTATCTGCTTGTATTGATTCAGAAACTATTCCAGACACG

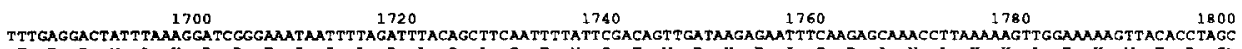

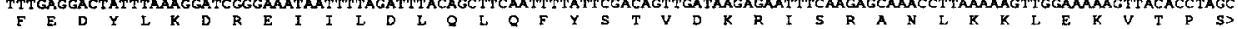

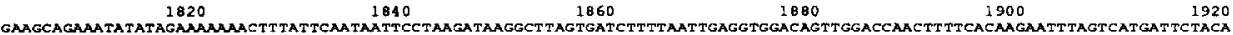

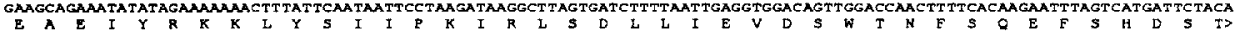
$\begin{array}{lccc}1940 & 1960 & 1980 & 2000 \\ \text { GGGAAACCGCCGAGTGAACAAGAAAGAAAATTATTTTGCTGCTTTGCTGGGTTAGGGATGAATATTGGTCTTGAAAAAATGGCCAATCAACTCCTGGAATTTCTTATTCTCAGTTA }\end{array}$ $20602020002120 \quad 2140 \quad 200$ GCCAATGCCAAACAATGGCGCTTTTATAAAGAAGCTCTGACTCGTGCTCAATCTGTTTTGGTTAATTATCAGTTAAAGCTTCCTGTTGCAGACTTTTGGGGTGAAGGAAAAACCACTGCT

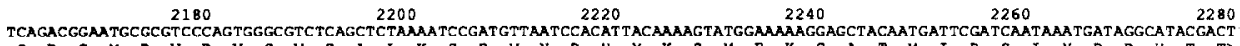

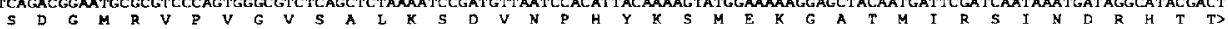
CATCATATCGAGGTTGCTTCAACTAATACAAGGGAAGCTACTCATACCCTEGATCGCCTACTTTATCATGAACAGATCTAGATATTGAGGAACATTTTACTGATACAAATCGGTATTCT H H I E V A S T T R E A T H T L D G L L Y H E T D I D I E E H F T D T N G Y S T GATCAGGTGTTTGGASTGACCGCATTACTAGGCTTTGATTTTGACCTCGCATCAGAAATATAAAAAAATCACAATTATTTTCTATCAAATCACGTTCCTACTACCCTAACTTATCAGAA

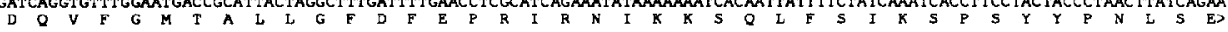
2540
GATATAAGCGGAAAATCAATGTAAAAATTATTGAAGMAAACTATGATGAATTAAACGAATCGCCTATTCGATTCAAACAGGAAAAGTATCTAGTTCTTTACTATTAGGAAGCTAGGC $\begin{array}{lllll}2660 & 2680 & 2700 & 2720 & 2740\end{array}$ TCATACGCACGTAAGATAGAGTAGCTCT TGCACTGAGAGAACTAGGTCGCATTGAAAGGAGCATT TTTATGATAGAT TATATTACAGATAGTGAGCTACGGCGAAGGATCACTCATGGA

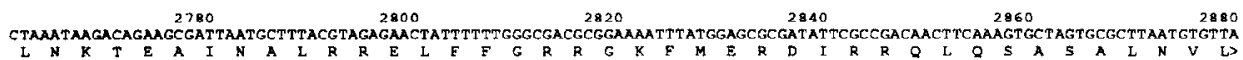
\begin{tabular}{lllll}
2900 & 2920 & 2940 & 2960 & 2980 \\
\hline
\end{tabular}

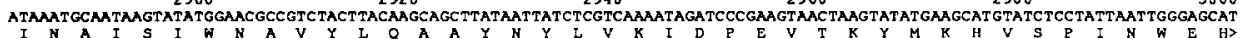
$\begin{array}{lllll}3020 & 3040 & 3060 & 3080 & 3100\end{array}$

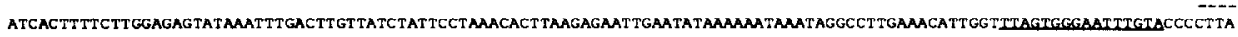

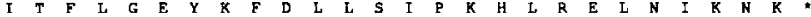
$\begin{array}{lllll}3140 & 3160 & 3180 & 3200 & 3220\end{array}$ TCGA

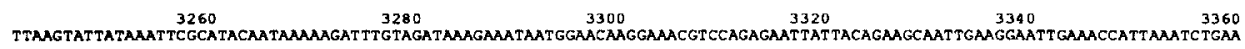
3380
TGCAGMAAMGAGTTTTTTGTMAATTGGAMAAACATGTTTACGTCTCAA

FIG. 1. The nucleotide sequence of tnpA (ORF5) of Tn917. The underlined regions correspond to the potential promoter, Shine Dalgarno (S. D.) site, and the inverted repeats that may represent a transcription terminator. The inferred amino acids of TnpA are shown in single-letter designations and are encoded from basepairs 160 to 3078 . The designation " $m p R^{*}$ " indicates the 3 ' terminus of $t n p R$, which is 16 bp 

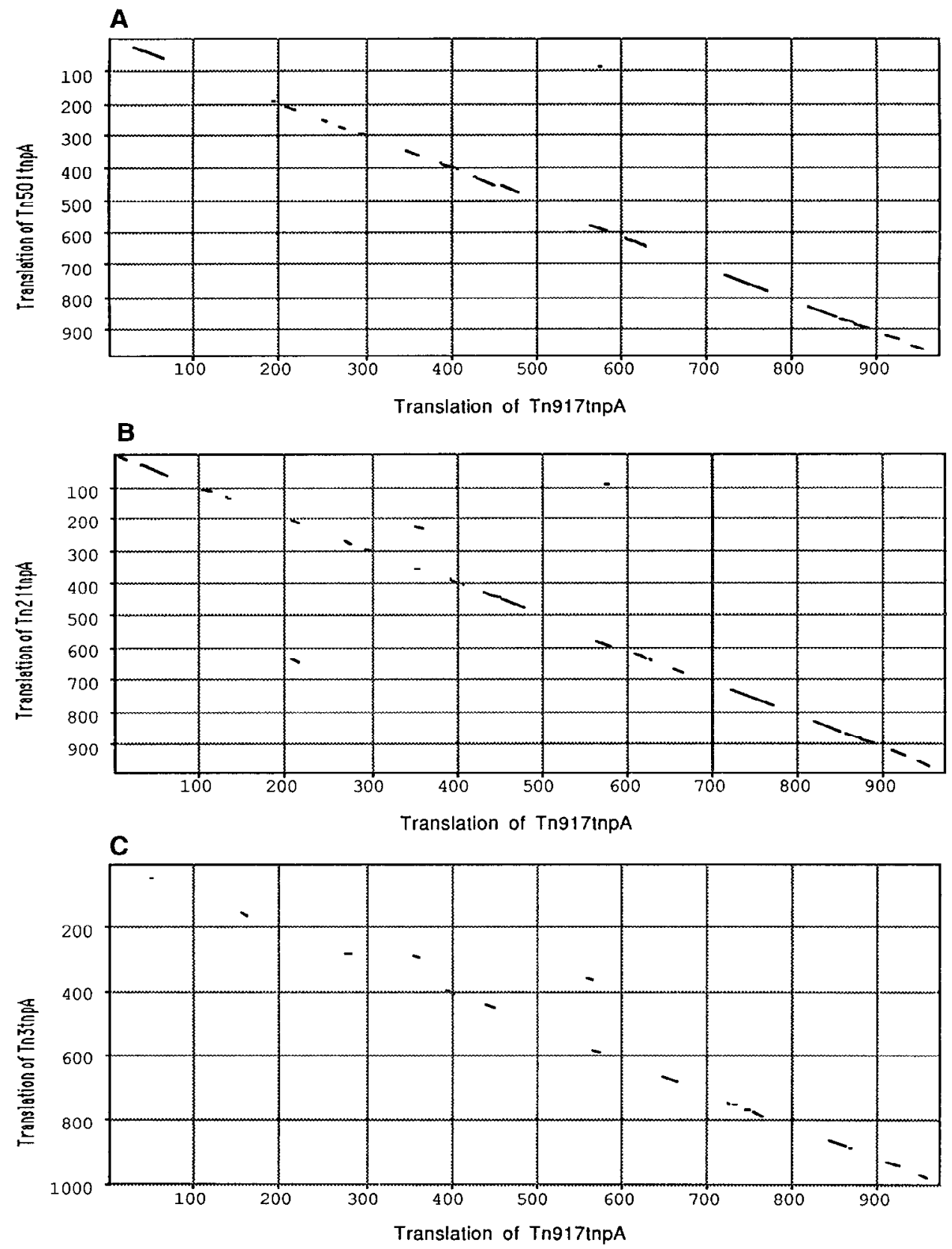

FIG. 2. Homology matrix plots comparing of $\operatorname{tnp} 4$ of $\operatorname{Tn} 917$ with $\operatorname{Tn} 501$ (A), $\operatorname{Tn} 21$ (B), and $\operatorname{Tn} 3$ (C). A MacVector (IBI) program was used to generate the comparisons. Each point reflects at least $50 \%$ homology within a 14-amino acid alignment with the corresponding protein.

upstream of the start site of $t n p A$. The dashed line designates the right-end 38-bp inverted repeat. Sequencing was performed utilizing a pBluescript (Stratagene, Inc.) clone (pAM3917R) of a segment from the pAD1::Tn917 derivative pAM938 (Ehrenfeld and Clewell, 1987). The clone contained the $3^{\prime}$ half of the transposon and adjacent pAD1 DNA. Overlapping nested deletions [ ExoIII/mung bean nuclease protocol (Stratagene, Inc.)] generated from both orientations were sequenced using the double-strand DNA procedure as described by Ausubel et al. (1987). A Sequenase kit from U.S. Biochemical Corp. was utilized. In some cases, specific synthetic primers made at the University of Michigan Biomedical Research Core Facility were used. 
$17 \%$, respectively. Homologies based on similarity are 53,50 , and $30 \%$, respectively.

The similarity of $\operatorname{Tn} 917$ with other $\operatorname{Tn} 3$ family transposons, with respect to both tnpR and $t n p A$, is consistent with a close evolutionary relationship. In this regard it is noteworthy that $\operatorname{Tn} 917$, which originated in the gram-positive $E$. faecalis, has been shown to transpose in a gram-negative (Escherichia coli) background (Kuramitsu and Casadaban, 1986).

\section{ACKNOWLEDGMENTS}

We thank N. Brown, J. Mahillon, and E. Murphy for pointing out the homologies between the ORF5 and ORF6 reading frames and tnpA of other Tn3-family elements, and N. Brown and D. Rouch for information on the likely position of certain frameshifts. This work was supported by USPHS Grants GM33956, AI10318, and DE02731.

\section{REFERENCES}

Ausubel, F. M., BRENT, R., Kingston, R. E., MOORE, D. D., Seidman, J. G., Smith, J. A., ANd Struhl, K. (1987). "Current Protocols in Molecular Biology." Wiley, New York.

Brown, N. L., Winnie, J. N., Fritzinger, D., AND PRIDMORE, R. D. (1985). The nucleotide sequence of the tnpA gene completes the sequence of the Pseudomonas transposon Tn501. Nucleic Acids Res. 13, 5657-5669.

EHRENFELD, E. E., AND Clewell, D. B. (1987). Transfer functions of the Streptococcus faecalis plasmid pAD1: Organization of plasmid DNA encoding response to sex pheromone. J. Bacteriol. 169, 3473-3481.

HefrRon, F. (1983). Tn 3 and its relatives. In "Mobile Genetic Elements" (J. A. Shapiro, Ed.), pp. 223-260. Academic Press, New York.
Heffron, F., McCarthy, B. J., Ohtsubo, H., and OHTSUBO, E. (1979). DNA sequence analysis of the transposon Tn3: Three genes and three sites involved in transposition of Tn3. Cell 18, 1153-1164.

KHAN, S. A., AND Novick, R. P. (1980). Terminal nucleotide sequence of $\mathrm{Tn} 551$, a transposon specifying resistance in Staphylococcus aureus: Homology with Tn3. Plasmid 4, 148-154.

Kuramitsu, H., and Casadaban, M. (1986). Transposition of the gram-positive transposon Tn917 in Escherichia coli. J. Bacteriol. 167, 711-712.

MURPHY, E. (1989). Transposable elements in grampositive bacteria. In "Mobile DNA" (D. E. Berg and M. M. Howe, Eds.), pp. 269-288. Am. Soc. Microbiol., Washington, DC.

Perkins, J., AND Youngman, P. J. (1984). A physical and functional analysis of $\operatorname{Tn} 917$, a Streptococcus transposon in the Tn 3 family that functions in Bacillus. Plasmid 12, 119-138.

SHaw, J., AND Clewell, D. B. (1985). Complete nucleotide sequence of macrolide-lincosamide-streptogramin B-resistance transposon $\mathrm{Tn} 917$ in Streptococcus faecalis. $J$. Bacteriol. 164, 782-796.

SherRATt, D. (1989). Tn 3 and related transposable elements: Site-specific recombination and transposition. In "Mobile DNA" (D. Berg and M. M. Howe, Eds.), pp. 163-184. Am. Soc. Microbiol., Washington, DC.

Tinoco, I., Borer, P., Dengler, B., LeVINe, M., UHLENBECK, O., CROTHERS, D., AND Gralla, J. (1973). Improved estimation of secondary structure in ribonucleic acids. Nature (London), New Biol. 246, 40-41.

Tomich, P., AN, F., AND Clewell, D. B. (1979). A transposon (Tn917) in Streptococcus faecalis that exhibits enhanced transposition during induction of drug resistance. Cold Spring Harbor Symp. Quant. Biol. 43, 1217-1221.

Tomich, P., AN, F., AND Ci.fwfi. I, D. R. (1980). Properties of erythromycin-inducible Tn 917 in Streptococcus faecalis. J. Bacteriol. 141, 1366-1374.

WARD, E., AND GRINSTED, J. (1987). The nucleotide sequence of the tnpA gene of $\operatorname{Tn} 21$. Nucleic Acids Res. 15, 1799-1806.

Communicated by Francis L. Macrina 